\title{
Improvement of methane hydrate detection using marine CSEM in the fictitious wave domain
}

\author{
Kei KUSUDA ${ }^{1}$, Hitoshi MIKADA ${ }^{1}$, Tada-nori GOTO ${ }^{1}$ and Junichi TAKEKAWA ${ }^{1}$ \\ ${ }^{1}$ Dept. of Civil and Earth Res. Eng., Kyoto University
}

\begin{abstract}
Marine controlled-source electromagnetic (CSEM) survey, one of the electro-magnetic (EM) sounding methods, is considered as a technique in practice for the exploration of hydrocarbon resources including methane hydrate $(\mathrm{MH})$. In the analysis of EM field acquired in CSEM survey, forward modeling is often used to evaluate sub-seafloor structure. In the forward modeling, transforming the diffusive Maxwell equation to a fictitious wave domain reduces CPU time (Mittet, 2010). In both the diffusive and fictitious wave domain, phase velocities of electromagnetic waves are a function of material properties, i.e., electric conductivity and magnetic permeability. In the fictitious wave domain, the difference in the phase velocity as a function of materials is exaggerated so that EM field could propagate through seawater with much slower apparent phase velocity compared to the other field propagating through sub-seafloor layers. However, such character of the fictitious wave domain has not been well exploited for the estimation of subsurface resistivity structure. In the present study, we examine whether the received waveforms in the fictitious wave domain could highlight $\mathrm{MH}$ responses better than in the diffusive domain. We conduct numerical simulations using a three-dimensional resistivity model composed of seawater and earth layers, and a thin $\mathrm{MH}$ zone of a rectangular shape. Our results show that the sensitivity to the $\mathrm{MH}$ response in the received waveform is improved in the fictitious wave domain. It is mainly due to the separation of EM waves travelling with different phase velocities through the sub-seafloor layers and seawater in the fictitious wave domain. We then tested to see if the transform from the diffusive domain (e.g., observed EM field) to the fictitious wave domain is possible or not for further utilization of the transform. As a result of the singular value decomposition method to achieve the transform, the transforming EM waves in the fictitious wave domain indicated that the sensitivity to MH becomes about twice as much than the original EM field in the diffusive domain.
\end{abstract}

\section{INTRODUCTION}

Methane hydrate $(\mathrm{MH})$ attracts increasing attention due to the recent growing demand of world-wide requirement of fossil fuels. While the base of most $\mathrm{MH}$ deposits is found clearly on seismic sections as cross-bedding events of the Bottom Simulating Reflector (BSR), the detailed structure of $\mathrm{MH}$ concentrated zones is not well delineated without any drilling. Furthermore, no visible BSR may not mean no presence of $\mathrm{MH}$ for some of MH deposits (Yuan and Edwards, 2000).

Marine controlled-source electromagnetic (CSEM) survey, one of the electro-magnetic (EM) sounding methods, is considered as a technique in practice for the exploration of hydrocarbon reservoirs including resistive Methane hydrate $(\mathrm{MH})$. This method has become firmly established as an important geophysical tool in the offshore environment (Constable, 2010). However, the spatial resolution of the method is lower than that of the seismic reflective survey, so that the improvement of its resolution is one of the great issues for MH exploration.

Recently, transforming the diffusive Maxwell equation to the fictitious wave domain has been developed to reduce computational time (Mittet, 2010). In this method, phase velocities of electromagnetic waves in the fictitious wave domain are a function of material properties, i.e., electric conductivity and magnetic permeability so that EM field could propagate through seawater with much slower apparent phase velocity compared to the other field propagating through sub-seafloor layers. However, such character of the fictitious wave domain has not been well exploited for the estimation of subsurface resistivity structure. We focus attention on this character and investigate whether the received waveforms in the fictitious wave domain could highlight $\mathrm{MH}$ responses better than in the diffusive domain.

In this study, we examined the possibility of $\mathrm{MH}$ detection with the transformed data from the diffusive domain to the fictitious wave domain. Transformation from the fictitious wave domain to 
the diffusive domain includes damping factor so that it is impossible to conduct inverse transform directly. We then experiment with singular value decomposition on the transform from the diffusive domain to the fictitious wave domain, and discuss the possibility of $\mathrm{MH}$ detection

\section{THEORY}

Our forward modeling algorism is described in detail in (Mittet, 2010). In the low-frequency limit relevant for the CSEM exploration, the quasi-static Maxwell equations in the diffusive time domain are

$$
\begin{gathered}
-\nabla \times \boldsymbol{H}(\boldsymbol{x}, t)+\boldsymbol{\sigma}(\boldsymbol{x}) \boldsymbol{E}(\boldsymbol{x}, t)=-\boldsymbol{J}(\boldsymbol{x}, t) \\
\nabla \times \boldsymbol{E}(\boldsymbol{x}, t)+\mu \partial_{t} \boldsymbol{H}(\boldsymbol{x}, t)=-\boldsymbol{K}(\boldsymbol{x}, t),
\end{gathered}
$$

where $\boldsymbol{E}$ and $\boldsymbol{H}$ are electric and magnetic vector fields, respectively. The source terms are electric current density $\boldsymbol{J}$ and magnetic current density $\boldsymbol{K}$. The conductivity tensor is $\boldsymbol{\sigma}$. The magnetic permeability $\mu$ is assumed to be isotropic and constant.

We introduced a fictitious dielectric permittivity tensor $\varepsilon^{\prime}$ from the conductivity tensor by

$$
\sigma(x)=2 \omega_{0} \varepsilon^{\prime}(x)
$$

where $\omega_{0}$ is an arbitrary parameter. The Maxwell equations in the fictitious time domain are

$$
\begin{gathered}
-\nabla \times \boldsymbol{H}^{\prime}\left(\boldsymbol{x}, t^{\prime}\right)+\boldsymbol{\varepsilon}^{\prime}(\boldsymbol{x}) \partial_{t^{\prime}} \boldsymbol{E}^{\prime}\left(\boldsymbol{x}, t^{\prime}\right)=-\boldsymbol{J}^{\prime}\left(\boldsymbol{x}, t^{\prime}\right), \\
\nabla \times \boldsymbol{E}^{\prime}\left(\boldsymbol{x}, t^{\prime}\right)+\mu \partial_{t^{\prime}} \boldsymbol{H}^{\prime}\left(\boldsymbol{x}, t^{\prime}\right)=-\boldsymbol{K}^{\prime}\left(\boldsymbol{x}, t^{\prime}\right),
\end{gathered}
$$

where $\boldsymbol{E}^{\prime}, \boldsymbol{H}^{\prime}, \boldsymbol{J}^{\prime}$ and $\boldsymbol{K}^{\prime}$ are related to $\boldsymbol{E}, \boldsymbol{H}, \boldsymbol{J}$ and $\boldsymbol{K}$ from the equation (1) and (2). The equation (3) is solved by the FDTD approach, with Yee's (1966) grids.

\section{(1) From the fictitious wave domain to the diffusive domain}

Mittet (2010) suggested the equation from the fictitious wave time domain to the diffusive frequency domain is

$$
\boldsymbol{E}(\boldsymbol{x}, \omega)=\int_{0}^{T} d t^{\prime} \boldsymbol{E}^{\prime}\left(\boldsymbol{x}, t^{\prime}\right) e^{-\sqrt{\omega \omega_{0} t^{\prime}}} e^{i \sqrt{\omega \omega_{0} t^{\prime}}}
$$

Note that this transform contains an exponential damping factor $e^{-\sqrt{\omega \omega_{0}} t^{\prime}}$ applied to the field calculated in the fictitious wave domain. Source function also transform in a similar way. Then the diffusive frequency domain Green's functions are calculated. With Green's functions at hand, electric and magnetic fields in the diffusive frequency domain resulting from electric or magnetic sources can be calculated. We obtain EM field in the diffusive time domain by a standard Fourier transform (Mittet, 2010).

\section{(2) From the diffusive domain to the fictitious wave domain}

We transform the observed data from the diffusive time domain to the diffusive frequency domain and obtain Green's function in the same way. Source functions in the fictitious wave domain transform to the diffusive frequency domain and we convolute these functions so that we can obtain the function in the diffusive frequency domain as a left side in the equation (4). Then we calculate the inverse transform the equation (4). As I mentioned before, we cannot calculated directly by an exponential damping factor. We suggest that censoring singular value decomposition method.

\section{RESULTS}

We calculate the electric field in the diffusive domain and the fictitious wave domain by the same source function and the same resistivity model and compare the MH layer response. The cross-section of the resistivity model is shown in Figure 1. We assumed the cuboids' $\mathrm{MH}$ layer sized $20 \mathrm{~m} \times$ $200 \mathrm{~m} \times 200 \mathrm{~m}$. A horizontal source dipole is $100 \mathrm{~m}$ and $1 \mathrm{~A}$. We assumed 14 receivers with intervals of $100 \mathrm{~m}$ between in line. The transmitter time function behaves as the first derivative of a Gaussian,

$$
\boldsymbol{J}(t)=2 \beta\left(t-t_{0}\right) \sqrt{\frac{\beta}{\pi}} e^{-\beta\left(t-t_{0}\right)^{2}},
$$

where $\beta=\pi f^{2}, t_{0}=\pi / f$ and $f=12.5 \mathrm{~Hz}$.

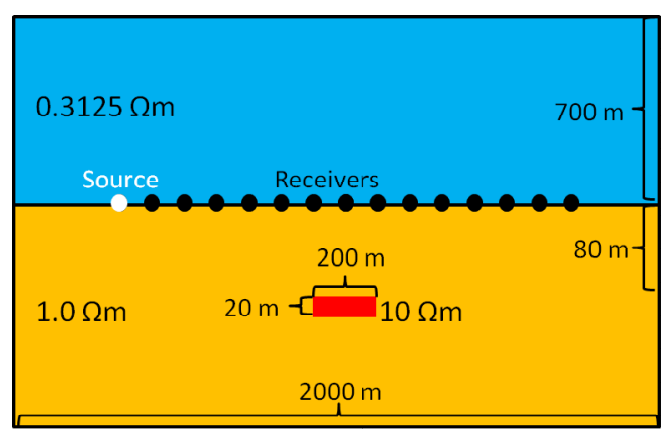


Figure 1: The cross-section of the 3D resistivity model, sub-seafloor structure including $\mathrm{MH}$

\section{(1) The diffusive domain}

In the diffusive domain, the inline electric field traveling through the sub-seafloor layers and seawater are arrived each receiver almost at the same time, so that the waveforms look single wave even if the offset became larger (Figure 2a). Strong $\mathrm{MH}$ response shows up only above the $\mathrm{MH}$ zone (Figure 2b). The maximum anomalous amplitude of the $\mathrm{MH}$ response is 7.9 percents at a source-receiver offset of $1000 \mathrm{~m}$.

\section{(2) The fictitious wave domain}

We found that the inline electric field is separated like a seismic propagation in the fictitious wave domain (Figure 3a). Here, the velocity of EM waves reduces effectively by resistivity of layers. As calculated from the equation (3), the velocity of the EM waves in seawater is $1767 \mathrm{~m} / \mathrm{s}$ and that of in seafloor is $10000 \mathrm{~m} / \mathrm{s}$. The electric field traveled through the seafloor and $\mathrm{MH}$ layers arrived first, and then the electric field traveled through the seawater arrived later. $\mathrm{MH}$ response shows up each receiver from the center of the $\mathrm{MH}$ zone (Figure $3 \mathrm{~b}$ ). The maximum anomalous amplitude of the $\mathrm{MH}$ responses is 31.6 percents at a source-receiver offset of $1000 \mathrm{~m}$.

\section{(3) Transform from the diffusive domain to the fictitious wave domain}

We examined to transform from the observed inline electric field at a source-receiver offset of $1000 \mathrm{~m}$ in the diffusive domain to the fictitious wave domain. In order to apply the singular value decomposition method, we need power spectrum with an accuracy of $10^{-22} \mathrm{~V} / \mathrm{m}$. However, it is impossible because the real data obtains noise signals. Constable (2010) indicates that noise floor for the electric field is approximately $10^{-15} \mathrm{~V} / \mathrm{Am}^{2}$. Here, we assumed the source dipole with length of $100 \mathrm{~m}$ and amplitude of $1 \mathrm{~A}$; referring the Constable (2010), we can use the observed data larger than $10^{-13} \mathrm{~V} / \mathrm{m}$ after transforming diffusive frequency domain, then we extrapolate the power spectrum from calculated one in the resistivity model without MH layers.

As a result of the singular value decomposition method for transformation, we achieved the separation of the electric waves through the sub-seafloor and seawater (Figure 4). The transforming EM waves in the fictitious wave domain indicated that the sensitivity to $\mathrm{MH}$ a)

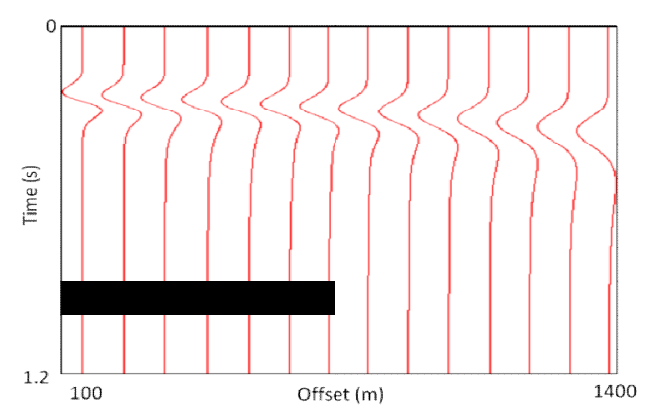

b)

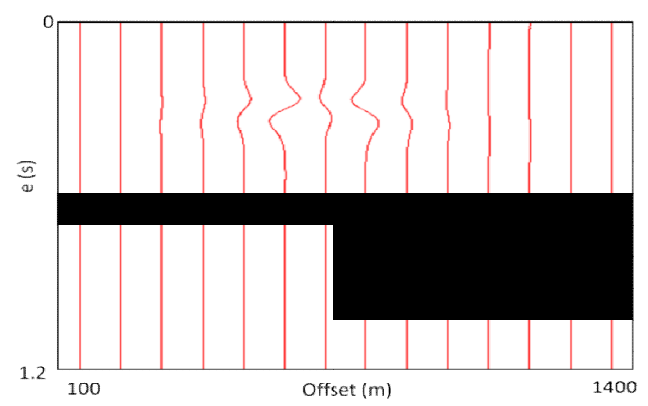

Figure 2: a) Traces of inline diffusive electric field at the 14 receivers. In order to confirm the waveforms, each amplitude range is normalized. b) The differences between responses with and without $\mathrm{MH}$ of inline diffusive electric field at the 14 receivers. The range of amplitude is from $1.0 \times$ $-10^{-12}$ to $1.0 \times 10^{-12} \mathrm{~V} / \mathrm{m}$.

a)

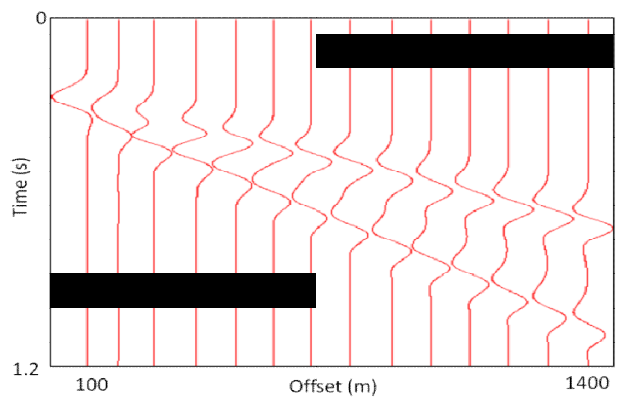

b)

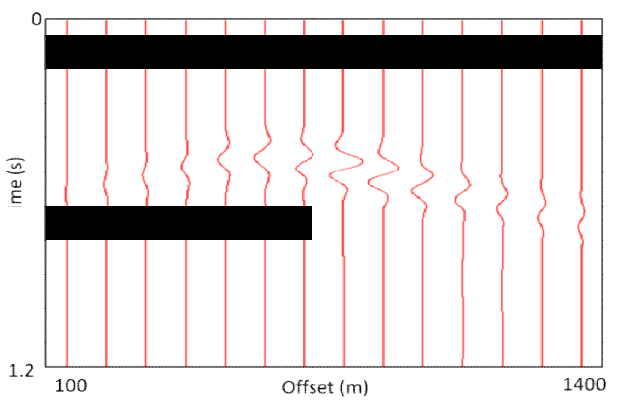

Figure 3: a) Traces of inline fictitious electric field at the 14 receivers. In order to confirm the Waveforms, each amplitude range is normalized. b) The differences between responses with and without $\mathrm{MH}$ of inline fictitious electric field at the 14 receivers. The range of amplitude is from $2.0 \times$ $-10^{-8}$ to $2.0 \times 10^{-8} \mathrm{~V} / \mathrm{m}$. 
calculated as 15.4 percents, which is about twice as much than the original EM field in the diffusive domain.

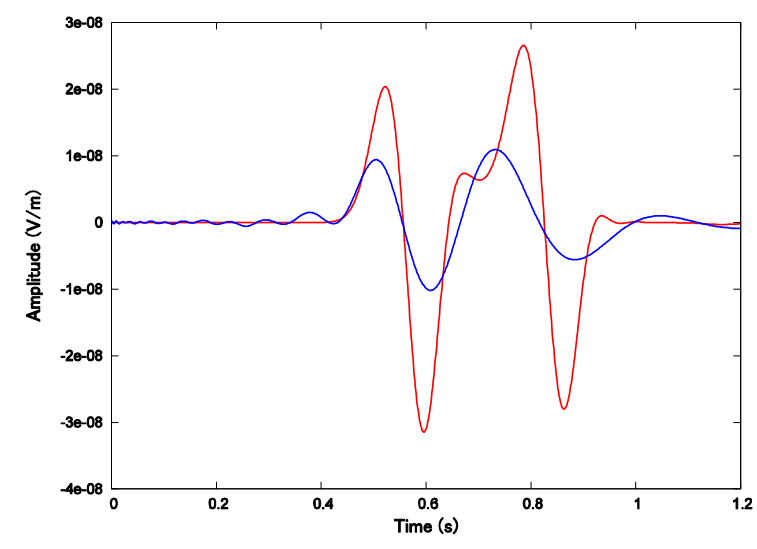

Figure 4: Inline fictitious electric field at a source-receiver offset of $1000 \mathrm{~m}$. Red: from model including $\mathrm{MH}$ layer in the fictitious wave domain. Blue: Electric field transformed from the diffusive domain to the fictitious wave domain

\section{DUSCUSSIONS}

Our results indicate that the EM wave propagation in the fictitious wave domain improves the $\mathrm{MH}$ response by separating the waves through the sub-seafloor and seawater. It is effective to analysis in the fictitious wave domain if we can transform the observed EM field in the diffusive domain to the fictitious wave domain. We achieved to separate the electric field and improve the $\mathrm{MH}$ response using singular value decomposition method to transform the diffusive domain to the fictitious wave domain. However, that waveform is rather smooth and decreases the sensitivity to $\mathrm{MH}$. We need to improve the accuracy of inverse transforming equation (4).

We also need to consider the effect of the noise floor. Although we used $10^{-13} \mathrm{~V} / \mathrm{m}$ as the cutoff value of inferred spectrum, the amplitude is the same order of the noise floor. We found that more realistic noise included in the observed EM field strongly disturbed the transformation to the fictitious wave domain. We should consider the way to reduce the noise level at least ten times, so that we are trying to modify our way of the transformation including the extrapolation of the power spectrum for overcoming the noise level.

We also examined if the observed EM data could be transformed back and forth between the fictitious wave domain and the diffusive domain using a numerical scheme. Employing a singular value decomposition method, we achieved the separation of the electric waves into waves traveled through the sub-seafloor, seawater, and $\mathrm{MH}$ concentrated zone. Although the sensitivity to $\mathrm{MH}$ layers is improved, the data transformed by this method does not exactly fit the received data calculated in the fictitious wave domain. After careful selection of the maximum condition number of the matrix for the transform, we observed the improvement in the recognition of $\mathrm{MH}$ response that become about twice as much as the original EM field in the diffusive domain. Although the transformation could be a powerful tool to interpret $\mathrm{MH}$ distribution, we need to consider how the transform could be stabilized numerically.

\section{CONCLUSIONS}

In the present study, we hypothesize that the transformation between the diffusive and the fictitious wave domain could be utilized to improve the $\mathrm{MH}$ response. We examined our hypothesis using 3-D numerical simulation. We confirmed that the difference in the apparent phase velocities of EM waves in the fictitious wave domain is exaggerated than in the diffusive domain so that the arrivals of EM waves propagated through different materials could readily be distinguished from each other. The method would improve the identification of $\mathrm{MH}$ response by wave separation.

\section{REFERENCES}

1) Collett, T.S., Lewis, R., and Uchida, T., 2000, Growing Interest in Gas Hydrates: Schlumberger Oilfield Review, 12 (2), 42-57.

2) Constable, S., 2010, Ten years of marine CSEM for hydrocarbon exploration: Geophysics, 75 (5), P75A67-75A81.

3) de Ryhove, S. de la Kethulle, J. P. Morten, and K. Kumar., 2013, Efficient 3D MT Inversion Using Finite-difference Time-domain Modelling: 75th EAGE Conference \& Exhibition incorporating SPE EUROPEC 2013.

4) Mittet, R., 2010, High-order finite-difference simulations of marine CSEM surveys using a correspondence principle for wave and diffusion fields: Geophysics, 75 (1), F33-F50.

5) Weitemeyer, K.A., Constable, S., and Trehu, A.M. (2011): A marine electromagnetic survey to detect gas hydrate at Hydrate Ridge, Oregon, Geophys. J. Int., doi: 10.1111/j.1365-246X.2011.05105.x.

6) Yee, K. S., 1966, Numerical solution of initial boundary value problems involving Maxwell's equations in isotropic media: IEEE Transactions on Antennas and Propagation 14 (3), 302-307. 
7) Yuan, J., and R. N. Edwards., 2000, The assessment of marine gas hydrates through electronic remote sounding: hydrate without a BSR?: Geophysical. Res. Lett., 27(16),

2397-2400. 